\title{
Estudo de parâmetros físicos, químicos e hídricos de um Latossolo Amarelo, na região Amazônica.
}

\author{
Jean Dalmo de Oliveira MARQUES ${ }^{1}$, Paulo Leonel LIBARDI ${ }^{2}$, Wenceslau Geraldes TEIXEIRA ${ }^{3}$, Ana Maria REIS ${ }^{4}$
}

\begin{abstract}
RESUMO
Estudou-se os atributos físicos, hídricos e químicos de um Latossolo sob sistema de agroflorestal na região Amazônica. O solo foi descrito morfologicamente e amostras com estrutura deformada foram coletadas para caracterização física e química. A análise das propriedades hidráulicas foi realizada a partir de amostras indeformadas coletadas a cada camada de $0,10 \mathrm{~m}$ até $1,00 \mathrm{~m}$ de profundidade com 5 repetições para determinação da condutividade hidráulica do solo saturado $\left(\mathrm{K}_{\mathrm{o}}\right)$ pelo método do permeâmetro de carga decrescente e determinação das curvas de retenção $\left(\phi_{\mathrm{m}}(\theta)\right)$, utilizando as tensões de 1, 2 e $4 \mathrm{kPa}$ (funis de placa porosa) e 10, 30, 50, 100, 500 e $1500 \mathrm{kPa}$ (câmaras de pressão com placa porosa). As demais análises foram realizadas até $1,50 \mathrm{~m}$ de profundidade. Os valores de Ko nas camadas apresentaram grande homogeneidade estando compreendido entre $2,22 \mathrm{a} 3,20 \mathrm{~cm} \mathrm{dia}^{-1}$, sendo relacionados com variação da densidade do solo e porosidade. A retenção hídrica demonstra que o solo tem a capacidade de reter elevada quantidade de água, mesmo quando submetido a elevados potenciais, indicando uma baixa disponibilidade para as plantas. As curvas de retenção não apresentaram um bom ajuste pela equação de van Genuchten, sugerindo uma distribuição de poros bimodal.
\end{abstract}

\section{PALAVRAS-CHAVE}

Condutividade hidráulica saturada, curva de retenção, atributos do solo, latossolo.

\section{Study of physical, chemical and bydric parameter of a xanthic ferralsol in the Amazon region.}

\begin{abstract}
The study of physical, bydric and chemical attributes of a Oxisol under the policultivation system in the Amazon region were studied. The soil was described morphologicaly and deformed structure samples were collected for physical and chemical characterization. The analyses of hydraulic properties was made from underformed samples collected in each $0.10 \mathrm{~m}$ soil layer up to $1.00 \mathrm{~m}$ depth with 5 replicates for the determination of hydraulic conductivity of satured soil $\left(K_{\sigma}\right)$ by the falling decreasing head permeameter method and determination of the retention curve $\left(\phi_{m}(\theta)\right)$, using the tensions of 1, 2 and $4 \mathrm{kPa}$ (porous plate funnels) and 10, 30, 50, 100, 500 and $1500 \mathrm{kPa}$ (porous plate pressure cells). Other analysis were made up to $1.50 \mathrm{~m}$ depth. The Ko values in the layers presented great bomogeneity between 2.22 and $3.20 \mathrm{~cm}$ day-1, related with the soil density variation and porosity.The hydric retention shows that the soil has the capacity to retain a great quantity of water, even when submitted to bigh potentials, indicating a low availability to the plants. The retention curves did not present a good adjustment by van Genuchten equation, suggesting a bimodal porous distribution.
\end{abstract}

\section{KEY WORDS}

Saturated bydraulic conductivity, retention curve, soil attributes, Oxisol.

\footnotetext{
${ }^{1}$ Universidade do Estado do Amazonas/UEA. Centro de Estudos Superiores de Tabatinga, Avenida da Amizade, $\mathrm{n}^{\circ}$ 74, Centro, CEP: 69.640.000, Tabatinga - AM. E-mail: jmarques@uea.edu.br

${ }^{2}$ Departamento de Ciências Exatas/ESALQ/USP, Av. Pádua Dias, n¹1, C.P. 09, CEP: 13418-900, Piracicaba, SP. E-mail: pllibard@carpa.ciagri.usp.br. Bolsista CNPq

${ }^{3}$ Embrapa - Amazônia Ocidental (Centro de Pesquisa Agroflorestal da Amazônia Ocidental - Manaus - AM - Caixa Postal: 319, CEP: 69011-970. E-mail: lau@cpaa.embrapa.br

${ }^{4}$ Universidade do Estado do Amazonas/UEA. Centro de Estudos Superiores de Tabatinga, Avenida da Amizade, n 74, Centro, CEP: 69.640.000, Tabatinga - AM. E-mail: amreis@uea.edu.br
} 


\section{ACTA \\ AMAZONICA}

ESTUDO DE PARÂMETROS FÍSICOS, QUÍMICOS E HIÍDRICOS

DE UM LATOSSOLO AMARELO, NA REGIÃO AMAZÔNICA

\section{INTRODUÇÃO}

A substituição da floresta por culturas agrícolas causa inúmeras mudanças nos atributos do solo que, em muitos casos, conduzem a perdas da produtividade. Nos últimos anos, os sistemas agroflorestais têm sido apontado como uma alternativa de recuperação e aproveitamento das áreas degradadas ou abandonadas na Amazônia.

É comum nas florestas tropicais a produção agrícola decrescer drasticamente em poucos anos após o desmatamento da floresta primária. Tal situação é atribuída a diversos fatores edáficos como pequena reserva de nutrientes, toxicidade de alumínio, baixa CTC, redução na infiltração da água no solo, redução da macroporosidade e destruição da estrutura do solo (Corrêa, 1985; Chauvel et al., 1991).

Estudos dos atributos do solo decorrentes da ação diferenciada dos processos de manejo adquirem grande importância na análise do comportamento das plantas cultivadas. Do ponto de vista prático é de grande interesse econômico o estudo dessas alterações, pois assumem relevante importância na recuperação ou manutenção do seu potencial agrícola (Fernandes, 1982).

$\mathrm{Na}$ Amazônia brasileira os trabalhos existentes até o momento tem demonstrado a viabilidade de utilização dos consorciamentos de espécies nativas como uma forma de recuperar áreas abandonadas (Zech et al., 1997; Teixeira, 1997).

Assim, o presente estudo objetiva caracterizar informações sobre atributos físicos, químicos e, principalmente, hídricos de um perfil de solo sob um sistema agroflorestal com o intuito de contribuir para o conhecimento básico e uso racional das terras da Amazônia.

\section{MATERIAL E MÉTODOS}

\section{Caracterização da área experimental}

O presente trabalho foi conduzido em uma área de terra firme na Estação Experimental do Centro de Pesquisa Agroflorestal da Amazônia Ocidental (Embrapa Amazônia Ocidental), localizada a 2 $2^{\circ} 52^{\prime}$ de latitude sul, 59 $59^{\circ}$ ' de longitude oeste, a uma altitude de $50 \mathrm{~m}$, no $\mathrm{km} 30$, da rodovia AM-010. As médias das temperaturas máximas e mínimas mensais são de $32^{\circ} \mathrm{C}$ e $22^{\circ} \mathrm{C}$, respectivamente. Segundo a classificação de Koppen o clima da região é do tipo Afi, caracterizado por apresentar precipitação média anual de $2420 \mathrm{~mm}$, com um máximo de $295 \mathrm{~mm}$ mensais, de março a abril, e um mínimo de $105 \mathrm{~mm}$ mensais, de agosto a setembro (EMBRAPA, 1984).

A área estudada faz parte de uma parcela experimental de $48 \times 32$ m constituinte de um sistema agroflorestal (SAF) composto por Cupuaçu (Theobroma grandiflorum), Urucum (Bixa orellana), Pupunha (Bactris gasipaes) e Castanha do Brasil (Bertboletia excelsa), tendo a Puerária (Pueraria phaseoloides). Há 11 anos a floresta primária foi removida e a área foi cultivada com seringueira por 3 anos sendo a seguir abandona. A floresta secundária emergente foi derrubada e queimada, sendo implantado o SAF. Adubações sucessivas foram realizadas durante o decorrer do desenvolvimento vegetativo das espécies. O solo estudado é um Latossolo Amarelo Distrófico, extremamente profundo, apresentando baixa CTC, baixa atividade de argila e alta porosidade (Marques et a1., 1999).

\section{Amostragem do solo e determinações}

Abriu-se um perfil de solo próximo as espécies cultivadas na parcela, com dimensão de 2,5 m x 2,5 m x 3,0 m para a descrição morfológica (Lemos \& Santos, 1996) e caracterização física, química e hídrica do solo. Coletaram-se amostras com estrutura deformada ao longo dos horizontes pedogenéticos identificados, em sacos plásticos, com aproximadamente $0,3 \mathrm{~kg}$ de solo, para as determinações físicas e químicas até $1,5 \mathrm{~m}$ de profundidade. A densidade do solo $\mathrm{r}\left(\mathrm{kg} \mathrm{m}^{-3}\right)$ foi determinada a partir de amostras indeformadas, utilizando anéis volumétricos. A determinação da densidade dos sólidos $\mathrm{r}_{\mathrm{s}}\left(\mathrm{kg} \mathrm{m}^{-3}\right)$ foi realizada pelo método do picnômetro conforme metodologia descrita no manual de métodos de análise físicas da EMBRAPA (1997).

A análise granulométrica foi realizada pelo método do densímetro descrito por Gree \& Bauder (1986). Utilizaramse $50 \mathrm{~g}$ de solo dispersando-o com solução aquosa de hexametafosfato de sódio puro $\left(6 \mathrm{~kg} \mathrm{~m}^{-3}\right)$ e de hidróxido de sódio $\left(4 \mathrm{~kg} \mathrm{~m}^{-3}\right)$ por agitação durante 16 horas. A fração areia foi separada da suspensão por lavagem e peneiramento em peneiras com malha de $0,053 \mathrm{~mm}$

As análises químicas foram realizadas de acordo com EMBRAPA (1997).

\section{Caracterização hídrica}

A caracterização hídrica do solo foi baseada em duas propriedades hidráulicas: curva de retenção e condutividade hidráulica do solo saturado (Gardner, 1986; Libardi, 2000). A realização de estudos hídricos favorece informações importantes para um melhor manejo de solo na Amazônia, já que análises envolvendo essas propriedades ainda são escassas na região, sendo comum resultados dos atributos físicos e químicos.

\section{Curva de retenção}

Para o estudo da retenção de água no solo, utilizaramse amostras com estrutura indeformada, sendo coletado um total de 50 amostras. As amostras foram coletadas a cada $0,10 \mathrm{~m}$, até $1 \mathrm{~m}$ de profundidade. Utilizou-se um amostrador de solo tipo Uhland, com anéis volumétricos, de 0,05 $\mathrm{m}$ de altura e $0,04 \mathrm{~m}$ de diâmetro. Após coletados, esses anéis foram acondicionados em papel alumínio para evitar perdas de umidade, sendo preparados no laboratório. 


\section{ACTA \\ AMAZONICA}

ESTUDO DE PARÂMETROS FÍSICOS, QUÍMICOS E HÍDRICOS DE UM LATOSSOLO AMARELO, NA REGIÃO AMAZÔNICA
A saturação das amostras foi feita com água destilada e desaerada, aplicada com gotejador de Mariotte, levando 24 horas para a completa saturação (Moraes, 1991).

As amostras foram avaliadas, utilizando-se mesa de tensão (EMBRAPA, 1997) para os potenciais mátricos (y): 1, 2 e 4 kPa e câmara de pressão para os seguintes (y): $10,30,50,100,500$ e $1500 \mathrm{kPa}$. Após entrarem em equilíbrio com a tensão, as amostras foram pesadas para a determinação da massa de solo úmido (m, kg), ressaturadas e recolocadas no aparelho para atingirem o próximo ponto de equilíbrio. Após a aplicação da tensão de $100 \mathrm{kPa}$, na câmara de pressão, as amostras indeformadas foram secas em estufa a $105^{\circ} \mathrm{C}$ por um período de 48 horas, para a determinação da massa de sólidos $\left(\mathrm{m}_{\mathrm{s}}, \mathrm{kg}\right)$. Com essa informação foi realizado o cálculo da umidade gravimétrica (U, $\mathrm{kg} \mathrm{kg}^{-1}$ ), umidade volumétrica ( $\mathrm{q}, \mathrm{m}^{3} \mathrm{~m}^{-3}$ ) e da densidade do solo ( $\mathrm{r}, \mathrm{kg} \mathrm{m}^{\text {- }}$ $\left.{ }^{3}\right)$. A determinação da umidade volumétrica nas tensões de 500 e $1500 \mathrm{kPa}$ foi realizada com as amostras com estrutura deformada, considerando que somente às baixas tensões o teor de água depende da estrutura do solo (Hillel, 1982). As amostras já secas após retiradas dos cilindros volumétricos foram desestruturadas com um rolo de madeira e passando em peneiras de $2 \mathrm{~mm}$, sendo em seguida colocadas em sacos plásticos devidamente identificados. A saturação das amostras deformadas foi realizada diretamente na placa porosa, lentamente. Em seguida, aplicou-se a tensão e após as amostras atingirem o ponto de equilíbrio, estas foram colocadas em recipientes para a obtenção da sua massa úmida e cálculo da umidade gravimétrica. A umidade volumétrica foi calculada baseada nos dados da densidade aparente do solo.

\section{Condutividade hidráulica}

A condutividade hidráulica foi determinada considerando as amostras coletadas até $1,00 \mathrm{~m}$ de profundidade, a cada $0,10 \mathrm{~m}$. A metodologia utilizada para a determinação da condutividade hidráulica do solo saturado foi a do permeâmetro de carga decrescente (Hendrickx, 1990; Youngs, 1991). Cilindros volumétricos com diâmetro $0,04 \mathrm{~m}$ e altura $0,05 \mathrm{~m}$ e de altura $0,04 \mathrm{~m}$ foram inseridos em um dispositivo previamente construído composto por um tubo de vidro transparente colocado em um módulo de encaixe (Figura 1).

As amostras foram saturadas conforme os procedimentos adotados na curva de retenção. Antes do ínicio das medidas foi delimitado dois pontos no tubo de vidro (Figura 1) denominados de $\mathrm{H}_{1}$ e $\mathrm{H}_{2}$ de forma que fosse possível acompanhar os seus respectivos tempos $\left(t_{1}\right.$ e $\left.t_{2}\right)$. Maiores detalhes sobre os procedimentos adotados para a determinação de $\mathrm{K}_{\mathrm{o}}$ podem ser visto em Marques (2001).
Dessa forma, a $\mathrm{K}_{\mathrm{o}}$ foi calculada pela seguinte equação: sendo:

$$
K_{o}=\frac{a L}{A \Delta t} \ln \left[\frac{H_{1}-H^{\prime}}{H_{2}-H^{\prime}}\right]
$$

$\Delta \mathrm{t}=\mathrm{t}_{2}-\mathrm{t}_{1}$ intervalo de tempo para o nível de água no tubo de vidro cair de $\mathrm{H}_{1}$ para $\mathrm{H}_{2}$;

$\mathrm{A}=$ área da seção transversal da coluna de solo;

$\mathrm{L}=$ comprimento da amostra de solo;

$\mathrm{a}=$ área da seção transversal do tubo de vidro onde se mede $\mathrm{H}$;

$\mathrm{H}_{1}$ e $\mathrm{H}_{2}$ representam potenciais totais da amostra, os quais foram fixados em dois pontos no tubo e mantidos constantes ao longo das medições;

$\mathrm{H}^{\prime}=$ carga hidráulica na superfície da amostra.

Considerando que a distribuição da condutividade hidráulica normalmente é log-normal (Logsdon \& Jaynes, 1996), a média da condutividade hidráulica saturada em cada seção do perfil estudado foi obtida a partir da média aritmética do logaritmo. A transformação logarítmica é necessária para reduzir as variações nos dados de $\mathrm{K}_{\mathrm{o}}$, mas os valores dos coeficientes de variação ainda podem permanecer elevados.

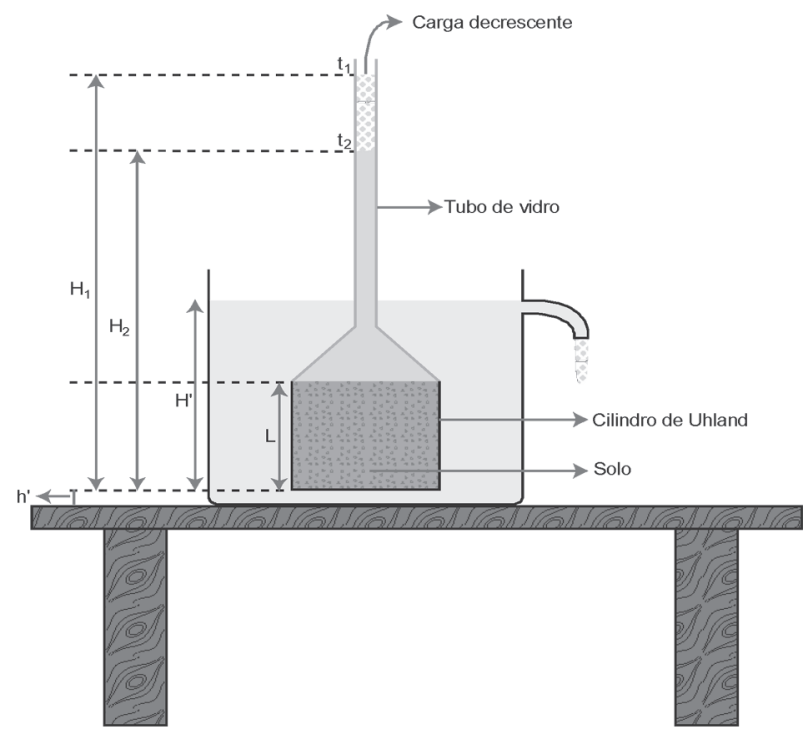

Figura 1 - Desenho esquemático do dispositivo utilizado na determinação da condutividade hidráulica do solo saturado (Libardi, 2000). sendo:

$\mathrm{Dt}=\mathrm{t}_{2}-\mathrm{t}_{1}$ intervalo de tempo para o nível de água no tubo de vidro cair de $\mathrm{H}_{1}$ para $\mathrm{H}_{2}$;

A = área da seção transversal da coluna de solo;

$\mathrm{L}=$ comprimento da amostra de solo;

$\mathrm{a}=$ área da seção transversal do tubo de vidro onde se mede $\mathrm{H}$;

$\mathrm{H}_{1} \mathrm{e} \mathrm{H}_{2}$ representam potenciais totais da amostra, os quais foram fixados em dois pontos no tubo e mantidos constantes ao longo das medições; H'= carga hidráulica na superfície da amostra. 


\section{Amostragem e análise estatística dos dados}

Um dos grandes problemas da utilização da estatística clássica é a necessidade de ter amostras independentes e dados que apresentam distribuição normal. Entretanto, em determinadas análises nem sempre ocorre tal situação, principalmente, quando se trata de estudos que envolvem retenção de água e condutividade hidráulica do solo.

A análise do comportamento hidráulico no solo estudado foi realizada com o auxilio de análise de variância e aplicação do teste de Tuckey ao nível de 5\% de probabilidade para observação das médias das umidades volumétricas em cada potencial mátrico $\left(\theta\right.$ vs $\left.\phi_{\mathrm{m}}\right)$ aplicado e condutividade até 1,00 m de profundidade, a cada $0,10 \mathrm{~m}$.

\section{Ajuste das curvas de retenção}

Com os valores das umidades associadas aos seus respectivos potenciais mátricos foram obtidos os ajustes conforme os parâmetros empíricos da equação proposta por van Genuchten (1980):

$$
\theta=\theta_{r}+\frac{\theta_{s}-\theta_{r}}{\left[1+\left(\alpha\left|\phi_{m}\right|\right)^{n}\right]^{m}}
$$

sendo $\theta$ a umidade volumétrica $\left(\mathrm{m}^{3} \mathrm{~m}^{-3}\right), \theta_{\mathrm{r}}$ a umidade residual $\left(\mathrm{m}^{3} \mathrm{~m}^{-3}\right), \theta_{\mathrm{s}}$ a umidade de saturação $\left(\mathrm{m}^{3} \mathrm{~m}^{-3}\right), \phi_{\mathrm{m}} \mathrm{O}$ potencial mátrico $(\mathrm{kPa})$ e a, m e n os parâmetros empíricos da equação. $\mathrm{O}$ ajuste das curvas foi feito com a utilização do programa SWRC desenvolvido por Dourado Neto et al. (1990). No ajuste, $\mathrm{q}_{\mathrm{s}}, \mathrm{q}_{\mathrm{r}}$, a e $\mathrm{m}$ foram estimados por regressão e $n$ foi considerado função de $m$, isto é, $m=1-1 / n$.

\section{Porosidade total, micro e macroporosidade}

A porosidade total foi calculada a partir da relação entre densidade do solo (r) e densidade dos sólidos $\left(\mathrm{r}_{\mathrm{s}}\right)$.
Considerando-se o limite de separação entre macro e microporos e referido para as tensões 6 e $10 \mathrm{kPa}$, considerou-se como porosidade capilar aquela que o solo apresenta depois de submetida a uma tensão de $10 \mathrm{kPa}$.

\section{RESULTADOS E DISCUSSÃO}

\section{Caracterização de alguns atributos físicos e químicos}

As tabelas 1, 2 e 3 apresentam os resultados analíticos das análises físicas e químicas. A análise granulométrica (Tabela 1) revelou homogeneidade na distribuição do tamanho das partículas ao longo do perfil, havendo um aumento do teor de argila com a profundidade $\left(0,59 \mathrm{~kg} \mathrm{~kg}^{-1}\right.$ a $0,73 \mathrm{~kg} \mathrm{~kg}^{-1}$ ), sendo classificado como de textura argilosa o horizonte superficial $(0-0,1 \mathrm{~m})$ e muito argilosa os demais. Os valores de densidade das partículas $\left(\mathrm{r}_{\mathrm{s}}\right)$ mostram pequenas variações, apresentando valor médio de $2560 \mathrm{~kg} \mathrm{~m}^{-3}$. Tal comportamento também foi observado para a densidade do solo (r) com um valor médio de $1060 \mathrm{~kg} \mathrm{~m}^{-3}$.

Analisando os resultados de análise química (Tabela 2), observa-se que o $\mathrm{pH}$ em $\mathrm{H}_{2} \mathrm{O}$ do LAd varia entre 4,07 a 4,23, não havendo muita variação entre os horizontes e caracterizando o caráter extremamente ácido deste solo (EMBRAPA, 1999).

O teor de matéria orgânica decresce em profundidade (Tabela 2), sendo o seu maior conteúdo encontrado na superfície devido à incorporação de resíduos vegetais provenientes do sistema agroflorestal. A manutenção dos teores de matéria orgânica no solo é um fator essencial para a conservação das propriedades físicas, químicas e produção das plantas em solos tropicais (Tisdall \& Oades, 1982; Zech et al. 1997). Apesar da matéria orgânica diminuir com a profundidade não foram verificadas grandes variações nos valores de densidade do solo (Tabela 1). O aumento gradativo do teor de argila (Tabela 1) pode ter compensado, de certa forma, a falta de variações expressivas da densidade do solo.

Tabela 1 - Distribuição granulométrica, densidade dos sólidos e do solo, e porosidade nos horizontes de um perfil de Latossolo Amarelo localizado na rodovia AM-010, Manaus.

\begin{tabular}{|c|c|c|c|c|c|c|c|c|c|}
\hline \multirow{2}{*}{ Prof.(m) } & \multirow{2}{*}{ Hor. } & \multirow{2}{*}{ AreiaTotal } & \multirow{2}{*}{ Silte } & \multirow{2}{*}{$\begin{array}{c}\text { Argila } \\
\text { Total }\end{array}$} & \multirow{2}{*}{$\begin{array}{c}\text { Densidade } \\
\text { do solo }\end{array}$} & \multirow{2}{*}{$\begin{array}{l}\text { Densidade } \\
\text { dos sólidos }\end{array}$} & \multicolumn{3}{|c|}{ Porosidade } \\
\hline & & & & & & & Total & Macro & Micro \\
\hline & & \multicolumn{3}{|c|}{. kg kg-1 - } & \multicolumn{2}{|c|}{ kg mºn } & \multicolumn{3}{|c|}{$m^{3} m^{-3} \ldots \ldots$} \\
\hline \multicolumn{10}{|c|}{ LATOSSOMO AMARELO Distrófico } \\
\hline $0,00-0,12$ & A & 0,21 & 0,20 & 0,59 & 1100 & 2560 & 0,56 & 0,14 & 0,42 \\
\hline $0,12-0,45$ & BA & 0,23 & 0,15 & 0,62 & 1060 & 2550 & 0,58 & 0,17 & 0,41 \\
\hline $0,45-0,75$ & Bw1 & 0,18 & 0,12 & 0,69 & 1020 & 2570 & 0,59 & 0,19 & 0,40 \\
\hline $0,75-1,00$ & Bw2 & 0,15 & 0,12 & 0,73 & 1080 & 2560 & 0,57 & 0,15 & 0,42 \\
\hline
\end{tabular}




\section{ACTA AMAZONICA}

Tabela 2 - Resultados das análises químicas de um perfil de Latossolo Amarelo localizado na rodovia AM-010, Manaus.

\begin{tabular}{|c|c|c|c|c|c|c|c|c|c|c|c|c|c|c|c|}
\hline \multirow{3}{*}{ Prof. (m) } & \multirow{3}{*}{ Hor. } & \multicolumn{3}{|c|}{$\mathrm{pH}$} & \multirow{2}{*}{ M.O. } & \multirow{2}{*}{$\mathbf{P}$} & \multirow{2}{*}{$\mathbf{K}^{+1}$} & \multirow{2}{*}{$\mathrm{Ca}^{+2}$} & \multirow{2}{*}{$\mathrm{Mg}^{+2}$} & \multirow{2}{*}{$\mathrm{Al}^{+3}$} & \multirow{2}{*}{$\mathrm{H}^{+} \mathrm{Al}$} & \multirow{2}{*}{ SB } & \multirow{2}{*}{$\mathbf{T}$} & \multirow{2}{*}{$\mathrm{m}$} & \multirow{2}{*}{ V } \\
\hline & & $\mathrm{H} 2 \mathrm{O}$ & $\mathrm{KCl}$ & C & & & & & & & & & & & \\
\hline & & 1120 & nel & & $\mathrm{g} \mathrm{kg}^{-1}$ & $\mathrm{mg} \mathrm{dm^{-3 }}$ & $-\cdots$ & $\cdots$ & $\cdots-\cdots$ & nolc kg & $s^{-1}-$ & & 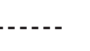 & -----\% & ----- \\
\hline $0,00-0,12$ & A & 4,1 & 3,9 & 3,5 & 31,0 & 12,0 & 4,6 & 8,5 & 6,7 & 21,2 & 90,2 & 19,8 & 110 & 52 & 18,0 \\
\hline $0,12-0,45$ & BA & 4,0 & 3,7 & 3,5 & 21,0 & 4,0 & 2,3 & 2,5 & 2,1 & 17,6 & 65,7 & 6,9 & 72,6 & 72 & 9,5 \\
\hline $0,45-0,75$ & Bw1 & 4,1 & 3,9 & 3,5 & 11,0 & 1,0 & 0,6 & 1,9 & 1,3 & 10,2 & 37,4 & 3,8 & 41,2 & 73 & 9,0 \\
\hline $0,75-1,00$ & Bw2 & 4,2 & 3,4 & 3,6 & 8,0 & 1,0 & 0,6 & 1,5 & 1,2 & 8,0 & 23,6 & 3,3 & 26,9 & 71 & 12,0 \\
\hline $1,00-1,50^{+}$ & Bw3 & 4,5 & 3,7 & 3,9 & 5,0 & 1,0 & 0,8 & 1,6 & 1,0 & 4,3 & 19,0 & 3,4 & 22,4 & 56 & 15,0 \\
\hline
\end{tabular}

M.O. = matéria orgânica; $\mathrm{T}=$ capacidade de troca de cátions $(\mathrm{S}+\mathrm{H}+\mathrm{Al}) ; \mathrm{m}=$ saturação por alumínio $(\mathrm{Al} / \mathrm{S}+\mathrm{Al}) .100 ; \mathrm{V}=$ saturação por bases $(100 . \mathrm{S} / \mathrm{T})$

Tabela 3 - Resultados do ataque sulfúrico em amostras de um perfil de Latossolo Amarelo localizado na rodovia AM-010, Manaus.

\begin{tabular}{|c|c|c|c|c|c|c|c|c|}
\hline Prof $(\mathrm{m})$ & Horizontes & $\mathrm{SiO}_{2}$ & $\mathrm{Al}_{2} \mathrm{O}_{3}$ & $\mathrm{Fe}_{2} \mathrm{O}_{3}$ & $\mathrm{Ti}_{2} \mathrm{O}_{2}$ & $\mathrm{MnO}$ & \multirow{2}{*}{$\mathrm{Ki}$} & \multirow{2}{*}{$\mathrm{Kr}$} \\
\hline Prot.(m) & Horizontes & & & $\mathrm{g} \cdot \mathrm{kg}^{-1}$ & & & & \\
\hline $0,00-0,12$ & A & 23,00 & 20,70 & 3,04 & 0,97 & 0,01 & 1,89 & 1,73 \\
\hline $0,12-0,45$ & BA & 29,10 & 26,52 & 3,75 & 1,20 & 0,01 & 1,87 & 1,71 \\
\hline $0,45-0,75$ & Bw1 & 30,60 & 27,23 & 4,04 & 0,17 & 0,01 & 1,91 & 1,74 \\
\hline $0,75-1,00$ & Bw2 & 34,60 & 28,86 & 4,15 & 1,43 & 0,01 & 2,04 & 1,87 \\
\hline $1,00-1,50^{+}$ & Bw3 & 35,70 & 30,80 & 4,06 & 1,39 & 0,01 & 1,97 & 1,82 \\
\hline
\end{tabular}

$\mathrm{Ki}=\left(\% \mathrm{SiO}_{2} / \% \mathrm{Al}_{2} \mathrm{O}_{3}\right) \cdot 1,7 ; \mathrm{Kr}=1,7 \% \mathrm{SiO}_{2} / \% \mathrm{Al}_{2} \mathrm{O}_{3}+0,6375 \% \mathrm{Fe}_{2} \mathrm{O}_{3}$

Os valores mais elevados de fósforo e soma de bases na superfície podem ser atribuídos aos maiores teores de matéria orgânica em superfície e adubações sucessivas, refletindo o efeito cumulativo da aplicação de adubos (Tabela 2).

A capacidade de troca de cátions ( $\mathrm{T}$ ) decresceu em profundidade, sendo que o valor de T variou de 110 a 26,9 $\mathrm{mmol}_{\mathrm{c}} \mathrm{kg}^{-1}$ de solo.

Ainda na Tabela 2, observa-se baixa saturação por base (V\%) e alta saturação por alumínio (m). Esta condição pode limitar o enraizamento das plantas em profundidade. Apesar da saturação por alumínio ser $>40 \%$ na camada de $0,2-0,4$ $\mathrm{m}$ e representar um fator restritivo ao crescimento das raízes (Hardy et al., 1990), percebe-se que as plantas cultivadas no sistema agroflorestal estudado apresentam grande capacidade de adaptação ao solo, desenvolvendo um sistema radicular superficial (pupunheira), lateral e moderado (Cupuaçuzeiro, Urucuzeiro) ou profundo (Castanheira), demonstrando grande adaptação às condições físicas e químicas.

Quando a atividade agrícola substitui uma floresta, o solo pode exaurir em poucos meses, ficando sujeito à erosão e lixiviação. Entretanto, os resultados dos atributos do solo demonstram que a consorciação de culturas agrícolas adaptadas à região, promovem o equilíbrio da reciclagem dos nutrientes que são extraídos do solo após decomposição da matéria orgânica. A produção do sistema é mantida pelo estoque de nutrientes fornecidas via decomposição da liteira. As espécies do SAF apresentam sistema radicular mais profundo que as culturas anuais, absorvem quantidades significativas de nutrientes do subsolo, que são depositados sobre a superfície via liteira, a partir da poda ou morte das raízes superficiais (Garrity et al., 1994). Já foi demonstrada a função das árvores perenes como reservatórios de nutrientes e o seu papel na reciclagem da biomassa em sistemas complexos (Burger, 1986).

A partir da Tabela 4, percebe-se a grande homogeneidade do perfil, apresentando na camada de $0,00-0,12 \mathrm{~m}$ a maior diferença quanto a cor e estrutura quando comparado com as demais.

\section{Condutividade hidráulica do solo saturado}

A Tabela 5 apresenta os resultados da condutividade hidráulica saturada e de seu logaritmo $\left(\log \mathrm{K}_{\mathrm{o}}\right)$. A análise estatística foi feita baseada nos valores obtidos a partir da transformação logarítmica dos dados, pois este parâmetro geralmente apresenta distribuição lognormal (Logsdon \& Jaynes, 1996).

Analisando os valores de $\log \mathrm{K}_{\mathrm{o}}$ obtidos ao longo das camadas, percebe-se que não houve diferença significativa entre as camadas, apresentando grande homogeneidade, 


\section{ACTA AMAZONICA}

Tabela 4 - Características morfológicas de um perfil de Latossolo Amarelo localizado na rodovia AM-010, Manaus.

\begin{tabular}{|c|c|c|c|c|c|c|}
\hline \multirow{2}{*}{ Hor. } & \multirow{2}{*}{ Prof (m) } & \multirow{2}{*}{ Textura } & \multirow{2}{*}{ Cor úmida } & Estrutura (1) & Consistência (2) & \multirow{2}{*}{ Transição } \\
\hline & & & & Tipo, Classe, Grau & Seca, Úmida, Molhada & \\
\hline \multicolumn{7}{|c|}{ LATOSSOLO AMARELO Distrófico } \\
\hline A & $0,00-0,12$ & argilosa & 10YR $4 / 3$ & $\begin{array}{l}\text { bl. sub., pequena } \\
\text { e média,moderada }\end{array}$ & $\begin{array}{l}\text { lig. dura, friável, } \\
\text { plástica e pegajosa }\end{array}$ & $\begin{array}{l}\text { gradual e } \\
\text { horizontal }\end{array}$ \\
\hline BA & $0,12-0,45$ & $\begin{array}{c}\text { muito } \\
\text { argilosa }\end{array}$ & 10YR 7/6 & $\begin{array}{l}\text { bl sub., pequena } \\
\text { e média, fraca }\end{array}$ & $\begin{array}{c}\text { macia, friável, } \\
\text { plástica e pegajosa }\end{array}$ & $\begin{array}{l}\text { difusa e } \\
\text { horizontal }\end{array}$ \\
\hline Bw1 & $0,45-0,75$ & $\begin{array}{c}\text { muito } \\
\text { argilosa }\end{array}$ & 10YR 7/8 & $\begin{array}{l}\text { bl sub., pequena } \\
\text { e média, fraca }\end{array}$ & $\begin{array}{l}\text { macia, friável, plástica } \\
\text { e muito pegajosa }\end{array}$ & $\begin{array}{l}\text { difusa e } \\
\text { horizontal }\end{array}$ \\
\hline Bw2 & $0,75-1,00$ & $\begin{array}{l}\text { muito } \\
\text { argilosa }\end{array}$ & 10YR 7/8 & $\begin{array}{l}\text { bl sub., pequena, } \\
\text { fraca }\end{array}$ & $\begin{array}{l}\text { macia, friável, plástica } \\
\text { e muito pegajosa }\end{array}$ & $\begin{array}{l}\text { difusa e } \\
\text { horizontal }\end{array}$ \\
\hline Bw3 & $1,00-1,50+$ & $\begin{array}{l}\text { muito } \\
\text { argilosa }\end{array}$ & 10YR $8 / 8$ & $\begin{array}{c}\text { bl. sub., pequena, } \\
\text { fraca }\end{array}$ & $\begin{array}{c}\text { macia, friável, plástica } \\
\text { e muito pegajosa }\end{array}$ & $\begin{array}{c}\text { difusa e } \\
\text { horizontal }\end{array}$ \\
\hline
\end{tabular}

(1) bl. sub. = bloco subangular; (2) lig. = ligeiramente

Tabela 5 - Condutividade hidráulica em diferentes camadas de um perfil de Latossolo Amarelo localizado na rodovia AM-010, Manaus, obtida em laboratório pelo método do permeâmetro de carga decrescente utilizando amostras indeformadas.

\begin{tabular}{lccr}
\hline \hline Camadas $(\mathrm{m})$ & Ko em cm dia ${ }^{-1}$ & Log Ko em cm dia-1 & Coeficiente de variação(\%) \\
\hline $0,00-0,10$ & 586,86 & 2,6240 a & 14,04 \\
$0,10-0,20$ & 1008,40 & 2,6549 a & 26,54 \\
$0,20-0,30$ & 761,12 & 2,5156 a & 27,03 \\
$0,30-0,40$ & 608,03 & 2,3986 a & 28,86 \\
$0,40-0,50$ & 2174,17 & 2,7798 a & 28,71 \\
$0,50-0,60$ & 2446,77 & 3,0630 a & 26,55 \\
$0,60-0,70$ & 2040,48 & 3,2051 a & 11,50 \\
$0,70-0,80$ & 1674,90 & 3,1725 a & 8,30 \\
$0,80-0,90$ & 659,70 & 2,5328 a & 26,52 \\
$0,90-1,00$ & 318,13 & 2,2229 a & 25,67 \\
\hline \hline
\end{tabular}

Médias obtidas a partir de 05 repetições

Médias seguidas de letras diferentes nas colunas indicam diferença significativa pelo teste de Tukey ao nível de 5\%.

Tabela 6 - Parâmetros de ajuste da equação de van Genuchten (1980) correspondentes as diferentes camadas de um perfil de Latossolo Amarelo localizado na rodovia AM-010, Manaus.

\begin{tabular}{ccccccccccc}
\hline \hline & \multicolumn{10}{c}{ Profundidades $(\mathrm{m})$} \\
\cline { 2 - 11 } Parâmetros & $\mathbf{0 , 1 0}$ & $\mathbf{0 , 2 0}$ & $\mathbf{0 , 3 0}$ & $\mathbf{0 , 4 0}$ & $\mathbf{0 , 5 0}$ & $\mathbf{0 , 6 0}$ & $\mathbf{0 , 7 0}$ & $\mathbf{0 , 8 0}$ & $\mathbf{0 , 9 0}$ & $\mathbf{1 , 0 0}$ \\
\hline $\mathrm{a}$ & 0,773 & 0,578 & 3,408 & 4,692 & 4,915 & 5,735 & 1,744 & 5,398 & 2,017 & 0,981 \\
$\mathrm{~m}$ & 0,014 & 0,011 & 0,014 & 0,080 & 0,105 & 0,091 & 0,079 & 0,133 & 0,027 & 0,131 \\
$\mathrm{n}$ & 1,014 & 1,012 & 1,014 & 1,087 & 1,117 & 1,100 & 1,085 & 1,154 & 1,028 & 1,151 \\
$\mathrm{qr}$ & $-1,736$ & $-1,780$ & $-1,719$ & 0,104 & 0,162 & 0,171 & 0,107 & 0,244 & $-0,377$ & 0,238 \\
qs & 0,566 & 0,545 & 0,522 & 0,601 & 0,614 & 0,595 & 0,588 & 0,605 & 0,569 & 0,580 \\
\hline \hline
\end{tabular}


O alto coeficiente de variação dessa propriedade é um dos principais inconvenientes na utilização da $\mathrm{K}_{\mathrm{o}}$ como parâmetro hidráulico. Dois fatores devem ser salientados quando se estuda essa propriedade em Latossolos argilosos na Amazônia Central.. O primeiro é volume da amostra, tendo que ser adaptado ao sistema poroso estudado, e conter um número representativo de cada classe de poros (Lauren et al., 1988). Quando uma amostra é retirada de uma camada, a continuidade vertical dos macroporos pode ser interrompida. O segundo, diz respeito à compactação da amostra durante o processo de extração que aliado ao preparo podem resultar na medição através de perturbações e selamento dos macroporos (Jarvis \& Meesing, 1995). Uma procedimento a ser seguido para uma melhor quantificação da condutividade hidráulica em solos com macroporos, como os amazônicos, é a coleta de amostras em cilindros grandes, minimizando as influências negativas citadas que as pequenas amostras exercem na determinação desse parâmetro. Além disso, o processo de extração requer um grande cuidado, já que o elevado teor de argila nesses solos eleva o risco de compactação.

Os valores dos coeficientes de variação apresentados na Tabela 4 podem ser devido as amostras terem sido tomadas bem próximas, tornando difícil a independência espacial e originando valores próximos, além da influência da quantidade excessiva de microporos. Tomasella \& Hodnett (1986), estudando a mesma classe de solo ao norte de Manaus, também encontraram alta variabilidade em profundidade, relacionando-a com a macroporosidade e estrutura . Entretanto, os resultados diferem dos aqui encontrados quanto à ordem de grandeza.

De modo geral, percebe-se que o $\log \mathrm{K}_{\mathrm{o}}$ foi sensível a pequenas variações na macroporosidade, apesar da comparação entre camadas não ter apresentado diferença estatística significativa, com exceção da camada $0,8 \mathrm{~m}$ $0,9 \mathrm{~m}$. Assim, verifica-se que a $\mathrm{K}_{\mathrm{o}}$ está relacionada com o volume de macroporos, bem como variações na estrutura (Ellies et al., 1997).

\section{Retenção de água no solo}

As umidades volumétricas foram relativamente altas em todos os potenciais mátricos avaliados, apresentando uma suave redução com o aumento da tensão. Uma provável explicação para essa tendência pode estar relacionada a maior proporção de microporos (aproximadamente 41\%) e menor de macroporos (17\%, Figura 1). Assim, os poros grandes perdem a água retida por capilaridade a potenciais elevados, enquanto que os pequenos, retém água até potenciais baixos adsorvida no sistema coloidal, ocasionando uma alta tensão caso existam em maior proporção (Hillel, 1971).
A pequena variação das umidades entre as tensões de 500 e $1500 \mathrm{kPa}$ podem ser explicadas pelos elevados teores de argila, pois conforme Hillel (1982), a textura e a superfície específica influenciam mais diretamente a retenção nessas tensões. A Tabela 6 demonstra que a água retida na capacidade de campo (tensão $30 \mathrm{kPa}$ ), situou-se entre 0,35 a $0,41 \mathrm{~m}^{3} \mathrm{~m}^{-3}$, estando o ponto de murcha permanente (tensão de $1500 \mathrm{kPa}$ ) entre 0,27 a $0,33 \mathrm{~m}^{3} \mathrm{~m}^{-}$ ${ }^{3}$, próximo aos encontrados por Correa, 1984 e Marques et al., 1999. Entretanto, Teixeira (2001), trabalhando com amostras indeformadas em solos da Amazônia, ressalta a influência do tempo da amostra na câmara de Richards para obtenção da umidade a $1500 \mathrm{kPa}$, salientando que nesses solos é necessário um maior tempo para atingir o ponto de equilíbrio.

Do ponto de vista agronômico, considerando os dados da Tabela 7, e adotando capacidade de campo como a umidade correspondente à tensão de $10 \mathrm{kPa}$, o solo estudado tem capacidade de reter um elevado teor de umidade, mesmo quando submetido a elevadas sucções, sugerindo uma baixa disponibilidade de água para as plantas sob estes potenciais.

Por outro lado, como as plantas cultivadas são adaptadas às condições locais, não há problemas de déficit hídrico, tendo água suficiente armazenada até a profundidade de 1,0 m. A partir dessa profundidade, há uma tendência de redução da água disponível devido à elevada energia de retenção, mas que em períodos secos na Amazônia, e dependendo da espécie, podem ser utilizados. Marques (1999), em amostragem realizada até $2,00 \mathrm{~m}$ de profundidade, revela esse comportamento. Plantas como a Castanheira e o Cupuaçuzeiro buscam a água em camadas mais profundas (Teixeira, 1997) demonstrando a redução do stress de água durante períodos críticos.

\section{Curva de retenção de água no solo}

Na Tabela 6 são apresentados os parâmetros de ajuste da equação de van Genuchten (1980). Analisando as curvas por horizontes (Figura 2), percebe-se que alguns pontos não apresentaram um bom ajuste pela equação. Uma explicação pode estar relacionada a distribuição da porosidade. Esses solos em condições naturais apresentam alta porosidade total (Côrrea, 1984), só que no espaço poroso do LAd predomina basicamente duas classes de poros, tendo muitos macroporos e quase que exclusivamente microporos (Tabela 1), evidenciando que a distribuição de poros que melhor se aplica é do tipo bimodal (Mallants et al., 1997) o que concorda com resultados encontrados por Chauvel et al. (1991) e Teixeira (2001) em solos dessa região. Ainda quanto à alta porosidade (Tabela 1), salienta-se a influência da Pueraria como cobertura de solo, que pode recuperar a porosidade alterada durante o desmatamento (Chauvel, 1987; Teixeira, 2001) o que aumenta o armazenamento da água, já que a sua disposição no solo reduz a evaporação (Marques, 2000). 
Tabela 7 - Valores médios obtidos de umidade volumétrica $\left(\mathrm{m}^{3} \mathrm{~m}^{-3}\right)$ em diferentes tensões, por camadas, de um perfil de Latossolo Amarelo Distrófico localizado na rodovia AM-010, Manaus.

\begin{tabular}{|c|c|c|c|c|c|c|c|c|c|c|}
\hline \multirow{3}{*}{ Camadas $(\mathrm{m})$} & \multicolumn{10}{|c|}{ Tensões aplicadas (kPa) } \\
\hline & 0 & 1 & 2 & 4 & 10 & 30 & 50 & 100 & 500 & 1500 \\
\hline & \multicolumn{10}{|c|}{-Umidade volumétrica $\mathrm{q}\left(\mathrm{m}^{3} \mathrm{~m}^{-3}\right)-\ldots$} \\
\hline $00,-0,10$ & 0,566 & 0,492 & 0,466 & 0,443 & 0,424 & 0,399 & 0,391 & 0,381 & 0,277 & 0,273 \\
\hline $\mathrm{DP}$ & 0,027 & 0,022 & 0,022 & 0,023 & 0,017 & 0,022 & 0,021 & 0,021 & 0,015 & 0,015 \\
\hline $\mathrm{CV}$ & 4,936 & 4,599 & 4,732 & 5,329 & 4,168 & 5,737 & 5,601 & 5,644 & 5,674 & 5,763 \\
\hline $0,10-0,20$ & 0,547 & 0,478 & 0,466 & 0,449 & 0,441 & 0,417 & 0,410 & 0,400 & 0,303 & 0,299 \\
\hline DP & 0,027 & 0,007 & 0,012 & 0,021 & 0,024 & 0,027 & 0,030 & 0,030 & 0,020 & 0,018 \\
\hline $\mathrm{CV}$ & 5,084 & 1,477 & 2,781 & 4,791 & 5,616 & 6,586 & 7,350 & 7,733 & 6,781 & 6,016 \\
\hline $0,20-0,30$ & 0,561 & 0,460 & 0,442 & 0,427 & 0,422 & 0,410 & 0,405 & 0,391 & 0,310 & 0,306 \\
\hline DP & 0,028 & 0,023 & 0,018 & 0,022 & 0,019 & 0,026 & 0,025 & 0,018 & 0,019 & 0,019 \\
\hline $\mathrm{CV}$ & 4,989 & 5,005 & 4,214 & 5,146 & 4,671 & 6,378 & 6,284 & 4,651 & 6,415 & 6,484 \\
\hline $0,30-0,40$ & 0,604 & 0,455 & 0,431 & 0,402 & 0,395 & 0,376 & 0,371 & 0,364 & 0,293 & 0,286 \\
\hline DP & 0,032 & 0,066 & 0,046 & 0,031 & 0,034 & 0,032 & 0,033 & 0,034 & 0,024 & 0,026 \\
\hline $\mathrm{CV}$ & 5,322 & 14,649 & 10,796 & 7,875 & 8,670 & 8,611 & 9,151 & 9,358 & 8,352 & 9,371 \\
\hline $0,40-0,50$ & 0,617 & 0,438 & 0,421 & 0,393 & 0,384 & 0,358 & 0,353 & 0,346 & 0,282 & 0,278 \\
\hline DP & 0,025 & 0,021 & 0,021 & 0,023 & 0,024 & 0,023 & 0,023 & 0,025 & 0,017 & 0,017 \\
\hline $\mathrm{CV}$ & 4,089 & 4,866 & 5,171 & 5,965 & 6,398 & 6,419 & 6,714 & 7,321 & 6,245 & 6,353 \\
\hline $0,50-0,60$ & 0,600 & 0,440 & 0,423 & 0,405 & 0,400 & 0,381 & 0,376 & 0,369 & 0,303 & 0,297 \\
\hline DP & 0,010 & 0,007 & 0,007 & 0,011 & 0,009 & 0,009 & 0,011 & 0,013 & 0,008 & 0,008 \\
\hline $\mathrm{CV}$ & 1,804 & 1,606 & 1,868 & 2,780 & 2,284 & 2,567 & 3,091 & 3,616 & 2,713 & 3,013 \\
\hline $0,60-0,70$ & 0,588 & 0,487 & 0,455 & 0,428 & 0,418 & 0,387 & 0,380 & 0,371 & 0,316 & 0,309 \\
\hline DP & 0,009 & 0,016 & 0,021 & 0,012 & 0,014 & 0,004 & 0,006 & 0,002 & 0,003 & 0,006 \\
\hline $\mathrm{CV}$ & 1,591 & 3,305 & 4,775 & 3,018 & 3,523 & 1,227 & 1,744 & 0,779 & 1,252 & 2,248 \\
\hline $0,70-0,80$ & 0,608 & 0,428 & 0,412 & 0,394 & 0,387 & 0,367 & 0,360 & 0,353 & 0,308 & 0,297 \\
\hline DP & 0,029 & 0,021 & 0,021 & 0,023 & 0,025 & 0,024 & 0,024 & 0,022 & 0,022 & 0,021 \\
\hline $\mathrm{CV}$ & 4,774 & 4,957 & 5,100 & 5,890 & 6,491 & 6,638 & 6,652 & 6,450 & 7,166 & 7,119 \\
\hline $0,80-0,90$ & 0.569 & 0.489 & 0.474 & 0.453 & 0.439 & 0.412 & 0.405 & 0.393 & 0.342 & 0.332 \\
\hline DP & 0.037 & 0.030 & 0.034 & 0.034 & 0.038 & 0.044 & 0.042 & 0.041 & 0.032 & 0.030 \\
\hline $\mathrm{CV}$ & 6,557 & 6,256 & 7,189 & 7,680 & 8,839 & 10,781 & 10,549 & 10,413 & 9,545 & 9,067 \\
\hline $0,90-1,00$ & 0.579 & 0.478 & 0.456 & 0.429 & 0.409 & 0.379 & 0.373 & 0.365 & 0.327 & 0.319 \\
\hline DP & 0.027 & 0.018 & 0.018 & 0.020 & 0.023 & 0.023 & 0.024 & 0.023 & 0.024 & 0.021 \\
\hline $\mathrm{CV}$ & 4,809 & 3,901 & 4,105 & 4,797 & 5,712 & 6,262 & 6,440 & 6,420 & 7,383 & 6,664 \\
\hline
\end{tabular}

DP = desvio padrão $\left(\mathrm{m}^{3} \mathrm{~m}^{-3}\right) ; \mathrm{CV}=$ coeficiente de variação $(\%) ; *$ Valores médios obtidos a partir de 5 repetições

\section{CONCLUSÕES}

O solo sobre o qual o sistema agroflorestal foi estabelecido apresenta bons atributos físicos e químicos.

Os valores de $\mathrm{K}_{\mathrm{o}}$ ao longo do perfil apresentaram grande homogeneidade, sendo mais sensíveis à variação da porosidade;

As curvas de retenção não apresentaram um bom ajuste pela equação unimodal de van Genuchten, indicando que para esses solos é necessário considerar os sistemas porosos inter e intragregados separadamente, sugerindo que a distribuição de poros seja a bimodal;

A retenção hídrica demonstra que o solo tem a capacidade de reter elevado teor de umidade, mesmo quando submetido a elevadas sucções, indicando nestes pontenciais baixa disponibilidade para as plantas. 


\section{ACTA \\ AMAZONICA}

\section{BIBLIOGRAFIA CITADA}

Burger, D.O. 1986. Uso da terra na Amazônia Oriental. In: Pesquisas sobre utilização e conservação do solo na Amazônia Oriental. Belém, EMBRAPA/CPATU/GTZ, p. 71-97. (Relatório Final do Convênio EMBRAPA/CPATU/GTZ).

Chauvel, A.; Lucas.; Y.; Boulet, R. 1987. On the genesis of the soil mantle of the region of Manaus, Amazônia Central, Brazil. Experientia. 43: 234-241.

Chauvel, A.; Grimaldi, M.; Tessier. 1991. Changes in soil porespace distribution following deforestation and revegetation: An example from the central Amazon basin, Brazil. Forest Ecology and Management. 38: 259-271.

Corrêa, J.C. 1984. Características físico-hídricas dos solos Latossolo Amarelo, Podzólico Vermelho Amarelo e Podzol Hidromórfico do Estado do Amazonas. Pesquisa Agropecuária Brasileira, 19:347-360

Corrêa, J.C. 1985. Características físicas de um latossolo amarelo muito argiloso (typic Acrorthox) do Estado do Amazonas, sob diferentes sistemas de preparo do solo. Pesquisa Agropecuária Brasileira, 20: 1381-1387.

Dourado Neto, D.; Jong Van Lier, Q.de.; Botrel, T. A.; Libardi, P. L. 1990. Programa para confecção da água no solo utilizando o modelo de Genuchten. Engenharia Rural, 1: 92-102

Ellies, A.; Grez, R.; Ramirez, C. 1997. La conductividad hidráulica en fase saturada como herramienta para el diagnostico de la estructura del suelo. Agro Sur, 5: 512-56.

EMBRAPA.1984. Centro Nacional de Pesquisa Agroflorestal. Boletim Agrometeorológico. Manaus, EMBRAPA - UEPAE (Documento, 6).

EMBRAPA. 1997. Manual de Métodos de Análise de Solo. $2^{\mathrm{a}}$ edição. rev. atual. Centro Nacional de Pesquisa de Solos. Rio de Janeiro, 212p. (EMBRAPA-CNPS. Documentos; 1).

EMBRAPA.1999. Sistema Brasileiro de Classificação de Solos. Centro Nacional de Pesquisa de Solos. Rio de Janeiro, EMBRAPA-CNPS, $412 \mathrm{p}$.

Fernandes, M. R. 1982. Alterações em propriedades de um Latossolo Vermelho Distrófico, fase cerrado decorrentes da modalidade de uso e manejo. Viçosa, 65p. Tese (Dissertação) - Universidade Federal de Viçosa.

Gardner, W. H. Water content. In: Klute, A. ed. Methods of soil analysis. Part I. Agronomy Mo nograph Séries $\mathrm{n}^{\circ}$ 9, Madison, Winconsin, Cap. 21, p. 493-544, 1986.

Garrity, D. P.; Lefroy, R. D. B.; Blair, G. J.; Craswell, E. T. 1994. The fate of organic matter and nutrients in agroforestry systems. In: Soil organic matter management for sustainable agriculture. Workshop held in Ubon, Thailand. Proceedings n. 56. Anais, Canberra: ACIAR. p. 69 - 77.

Gree, G. W.; Bauder, J. C.1986. Particle-size analysis. In: Klute, A. (Ed). Methods of soil analysis, I. Physical and mineralogical methods. Madison. American Society of Agronomy, cap. 15, p. 383 -411.

Hardy, D. H.; Rapper, C. D.; Miner, G. S. 1990. Chemical restrictions of roots in ultisol subsoils lessened by long-term management. Soil Science Society of America Journal,. Madison, 54: 1657-1660.
Hendrickx, J. M. H. 1990. Determination of hydraulic soil properties In: Anderson, M. G. \& Burt, T. P. Process Studies in Hillslope Hydrology. England, cap. 3, p. 43-92.

Hillel, D. 1971. Soil and water. Physical principles and processes. New York, Academic Press, 288p.

Hillel, D. 1982. Introduction to Soil Physics. New York, Academic Press, 365p.

Jarvis, N. J. \& Messing, I. 1995. Near-saturated hydraulic conductivity in soils of contrasting texture measured by tension infiltrometers. Soil Science Society of America Journal, 59: p. 27-34.

Lemos, R. C.; Santos, R. D. dos. 1996. Manual de descrição e coleta de solo no campo. $3^{\mathrm{a}}$ ed. Campinas, SP:SBCS/CNPS, 83p.

Libardi, P. L. Dinâmica da água no solo. $2^{\mathrm{a}}$ edição. Piracicaba, 2000: o autor. $509 \mathrm{p}$.

Logsdon, S. D.; Jaynes, D. B. 1996. Spatial variability of hydraulic conductivity in a cultivated field at different times. Soil Science Society of America Journal, 60: 703-709.

Mallants, D.; Tseng, P. H.; Toride, N.; Timmerman, A.; Feyen, J. 1997. Evaluation of multimodal hydraulic functions in characterizing a heterogeneous field soil. Journal of Hydrology, 195:172-199.

Marques, Jean Dalmo., Teixeira, W. G., LEHMANN, Johannes Caracterização e Interpretação de Parâmetros físicoquímicos de um Latossolo amarelo, na região Amazônica. . In: XXVII Congresso Brasileiro de Ciência do Solo. 1999, Rio de Janeiro. Anais. Rio de Janeiro: Embrapa Solos, 1999. v. CD ROM. p. 1 - 3

Marques, Jean Dalmo., Teixeira, W. G., LEHMANN, Johannes Soil water availability as affected by the cover crop Pueraria phaseoloides in the Central Amazon. In: German-Brasilian Workshop on Neotropical Ecosystems, 2000, Hamburgo. Achievvements and prospects of cooperative research. Hamburgo: Universidade de Hamburgo, 2000. v.1

Marques, J. D. 2001. Horizontes pedológicos e sua relação com os horizontes hidráulicos do solo. Piracicaba, 86p. Tese (Dissertação) - Escola Superior de Agricultura Luiz de Queiroz, Universidade de São Paulo.

Moraes, S. O. 1991. Heterogeneidade hidráulica de uma terra roxa estruturada. Piracicaba, 141p. Tese (Doutorado) Escola Superior de Agricultura Luiz de Queiroz, Universidade de São Paulo.

Teixeira, W. G.; Schroth, G.; Faccin, O. P.; Huwe, B.; Zech, W. 1997. Avaliação de parâmetros físico-químicos e hídricos do solo como indicadores da recuperação de áreas degradadas na Amazônia Ocidental. In: Simpósio Nacional de Recuperação de áreas degradadas. Ouro Preto, Viçosa/ UFV, p. 166-174.

Teixeira, W. G. 2001. Land use effects on Soil Physical and Hydraulic Properties of a Clayey Ferralsol in the Central Amazon. University of Bayreuth. Bayreuth - Alemanhã Tese de Doutorado. 255p.

Tisdall, O. M.; Oades, J. M.1982. Organic matter and water-stable aggregates in soils. Journal of Soil Science, 33:141-163 
Tomasella, J.; Hodnett, M. G. Soil hydraulic properties and van Genuchten parameters for an Oxisol under pasture in Central Amazonia. In: Gash, J. H. C.; Nobre, C. A.; Roberts, J. M.; Victoria, R. L. Amazonian deforestation and climate. West Sussex, Jonh Wiley, 1996. p. 101 - 124.

Van Genuchten, M. TH. 1980. A closed form equation for predicting the hidraulic conductivity for unsatureted soils. Soil Science Society of American Journal, 44: 892-898.

Youngs, E.G.1991. Hydraulic conductivity of saturated soils. In: Smith, K. A.; Mullins, C.E. (Ed). Soil analysis: physical methods. New York: Marcel Dekker. cap. 4, p. 161-207.
Zech, W.; Senesi, N.; Guggenberger, G.; Kaiser, K.; Lehman, J.; Miano, T. M.; Miltner, A.; Schroth, G.1997. Factors controlling humification and mineralization of soil organic matter in the tropics. Geoderma, 79:117-161.

RECEBIDO EM 07/10/2002
ACEITO EM 03/05/2004 